\title{
Contribution à l'étude des rotations culturales : tentative d'utilisation d'un modèle
}

\author{
Maurice CABELGUENNE, Charles Allan JONES (*), Jean-Robert MARTY \& Hector QUINONES (**) \\ I.N.R.A., Station d'Agronomie, Centre de Recherches de Toulouse, B.P. 27, F 31326 Castanet Tolosan Cedex \\ $\left(^{*}\right)$ U.S.D.A.-A.R.S., P.O. BOX 6112, Temple Tx 76503, U.S.A. \\ (**) Consejo Nacional de Cienca y Technologica, 04515, Mexico, DF Mexique
}

RÉSUMÉ

\begin{abstract}
Les recherches concernant le fonctionnement des successions de cultures, conduites à différents niveaux d'intensification incitent à réaliser une synthèse des nombreux résultats expérimentaux, par l'utilisation de modèles de type global. Le modèle utilisé ici, élaboré par une équipe de chercheurs de l'Agricultural Research Service (U.S.D.A.-A.R.S.) perfectionné et validé à l'I.N.R.A.-Toulouse avec la collaboration du laboratoire de Biométrie, prend en compte les relations plante-sol-climat-techniques-successions de cultures. Cet article compare plusieurs années de résultats simulés à ceux mesurés sur un dispositif expérimental de longue durée. Les comparaisons portent notamment sur les bilans hydriques et de production pluriannuels et sur l'évolution des nitrates dans le sol pour des successions de cultures conduites à différents niveaux d'intensification. Des productions de blé et de cultures d'été sous différentes conditions de précédents culturaux et d'intensification sont aussi comparées. Les perfectionnements récents apportés au modèle lui permettent de simuler correctement des processus essentiels et d'obtenir des résultats avec une marge d'erreur acceptable. Ceci pourrait lui conférer des possibilités d'utilisation pour établir des diagnostics et aider à la prise de décision, par exemple l'irrigation des cultures d'été, et la fertilisation azotée du blé. Des progrès sont en cours, à partir de ce type de modèle, en collaboration avec le laboratoire de l'U.S.D.A.-A.R.S. de Temple (Texas) : simulation des stress eau et azote selon les stades physiologiques des cultures, de la fixation symbiotique des légumineuses d’hiver (pois et féverole).
\end{abstract}

Mots clés additionnels: Systèmes de culture, itinéraires techniques, intensification culturale, bilans hydriques, alimentation hydrique, bilans azotés, production. rendement.

Research concerning crop rotation at different levels of intensification can be assisted by the synthesis of experimental results using simulation models. The model here used, developed by a research team of the US agricultural research service (U.S.D.A.-A.R.S.) modified and validated by I.N.R.A.-Toulouse (Agronomy and Biometry laboratories), takes into account the relationships between crop-soil-climate-management activity-crop rotation. This paper compares simulated results with those measured over several years in a long-term field experiment. These comparisons concerned water balance, crop production and nitrogen evolution in the soil according to different crop rotations and levels of inputs. Simulated and measured results of winter wheat and summer crops were also compared in the same conditions. Following recent improvements, the model simulated the essential processes correctly and reproduced the measured results with an acceptable error. This agreement makes it possible to use the model as a decision aid for summer crop irrigation and for nitrogen fertilisation of wheat. The following aspects of the model are currently being improved in collaboration with the U.S.D.A.A.R.S. laboratory at Temple (Texas) : water and nitrogen stresses according to crop phenological stages, nitrogen fixation of winter legume crops (pea and horse bean).

Additional key words : Cropping system, management activity, water balances, water consumption, nitrogen balances, produce, yield.

\section{INTRODUCTION}

L'étude expérimentale de rotations culturales et la synthèse des résultats se heurtent à d'importants problèmes dus à la multiplicité des interactions solplante-climat-techniques et à l'aspect pluriannuel de cultures qui se succèdent sur une même parcelle.

C'est pourquoi nous avons tenté d'utiliser un modèle 
qui, s’appuyant sur les références expérimentales, permette de simuler le fonctionnement de successioris de cultures, et de constituer un outil généralisable à diverses conditions et situations.

La modélisation représente, en effet, un moyen privilégié pour organiser et synthétiser les nombreux résultats expérimentaux. Nombreux sont les modèles qui simulent par espèce l'élaboration de la matière sèche totale (JACKSON \& ARKIN, 1982; WILKINSON et al., 1983 ; JoNES et al., 1984, 1986 ; PEARSON et al., 1985), plus rares sont ceux qui intègrent les conditions culturales et la succession pluriannuelle des cultures (WHISLER et al., 1986) et c'est un modèle de ce type que nous avons choisi d'utiliser. Il s'agit d'EPIC (Erosion Productivity Impact Calculator, WILliams et al., 1984).

Ce modèle peut être considéré à la fois comme outil de synthèse et de recherches agronomiques, et comme aide à la prise de décision pour des interventions techniques. Nous avons déjà montré (CHARPENTEAU et al., 1986 ; Cabelguenne et al., 1986) qu'il a pu être globalement validé sur 10 rotations différentes conduites pendant 10 ans dans la région toulousaine avec des technicues courantes correspondant à un niveau moyen d'intensification. Notre objectif est ici de préciser des possibilités d'utilisation plus affinées, appliquées à des cultures et à des rotations conduites à différents niveaux d'intensification. Cet objectif plus ambitieux fera apparaître les limites du modèle, mais aussi les possibilités d'amélioration que nous tenterons de discuter.

\section{MATÉRIEL ET MÉTHODE}

\section{A. Rappel des caractéristiques du modèle}

\section{Les principes généraux}

Le modèle EPIC a été élaboré par un groupe de chercheurs de l'USDA-ARS. Ce modèle est capable: de simuler une succession de différentes cultures en calculant la croissance et la production de plusieurs espèces, en prenant en compte les itinéraires techniques (SEBILLOTTE, 1974) (travail du sol, fertilisation, irrigation, etc...), en décrivant les interrelations plante-sol-climat. Il a été installé, adapté et perfectionné à Toulouse.

Sans rentrer dans le détail des équations, nous résumons succinctement le principe du fonctionnement journalier du modèle dans la figure 1. Chaque partie du système sol-plante-atmosphère-technique est pris en compte à partir de sous-programmes du modèle. Ils concernent :

- l'hydrologie et les mouvements de l'eau dans le sol,

- l'évapotranspiration du système sol-plante,

- le climat,

- la matière organique (résidus de récolte et matière humique) et les éléments nutritifs (azote nitrique et acide phosphorique essentiellement),

- la croissance des différentes espèces, différenciées par des paramètres concernant les parties aériennes, les parties racinaires par des contraintes de croissance, et par l'activité symbiotique pour les légumineuses,

- les techniques (travail du sol, fertilisation, irrigation).
Précisons que la contrainte de croissance est appréhendée par les stress - jours (le modèle distingue les stress eau, azote, phosphore, température et croissance racinaire). Ces stress sont exprimés par un coefficient d'intensité a, variant de 0 (croissance nulle) à 1 (croissance potentielle). A la fin de la simulation apparaît, par mois, le nombre de stress $\cdot$ jours $=\Sigma(1-a) \cdot$ nombre de jours.

\section{Les données nécessaires au fonctionnement du modèle}

Elles sont présentées sur la figure 1. Précisons qu'en présence d'une légumineuse, EPIC simule les 2 voies d'alimentation azotée de cette espèce, la simulation de l'activité symbiotique ayant été élaborée à l'I.N.R.A.Toulouse pour le soja et doit se poursuivre pour le pois et la féverole. On peut ajouter que le modèle possède un "générateur de climat » (RICHARDSON, 1981).

\section{B. Les données qui permettent le perfectionnement et la validation du modèle}

La Station d'Agronomie de l'I.N.R.A. Toulouse a mis en place en 1983 un dispositif expérimental de longue durée pour étudier les effets de différents niveaux d'intensification culturale appliqués à des rotations renfermant des proportions variables de céréales et d'oléoprotéagineux (MARTY et al., 1981). Il s'agit de 10 rotations, renfermant 9 espèces et conduites à 3 niveaux d'intensification, qui offrent donc un large éventail exploratoire de conditions de production au cours d'années climatiques différentes, et sur lequel travaillent différents chercheurs français ou étrangers. Par ailleurs, des expérimentations plus détaillées concernant la physiologie du tournesol et du soja sont réalisées avec des conditions variées d'alimentation hydrique et azotée. A proximité de ce dispositif sont installés un parc climatique et des cases lysimétriques. Une partie des données recueillies sur ces installations expérimentales sert à perfectionner le modèle (utilisation de l'eau par les cultures, activité symbiotique par exemple) et à le valider.

Les mesures réalisées sur le terrain et utilisées pour la validation concernent d'une part le sol : teneur en eau, dessiccation, réhumectation, teneur en azote total, en azote nitrique, température à différentes profondeurs, densité apparente, et d'autre part la plante (sur les 9 cultures concernées, d'hiver : blé, orge, pois, féverole, colza, et d'été : maïs, tournesol, soja, sorgho), évolution dans le temps de l'indice foliaire et de la matière sèche totale (évolution de l'absorption des éléments minéraux), évolution de l'enracinement par observation endoscopique (MAERTENS, 1987), indice de récolte (rapport grain/matière sèche totale) quantités d'azote exportées et restituées.

Les résultats qui vont suivre ont fait l'objet d'une validation pas à pas selon les possibilités fournies par les expérimentations au champ. Ainsi, sur les diverses cultures ont été validés l'évolution des consommations en eau, la dessiccation du sol, les quantités de matière sèche totale produite et d'azote absorbé, les rendements et résidus de récolte et les restitutions en azote; par contre, certaines valeurs simulées n'ont pas encore été validées telles que l'influence du travail du sol sur la température, l'aération, l'infiltration de l'eau, la plus ou 

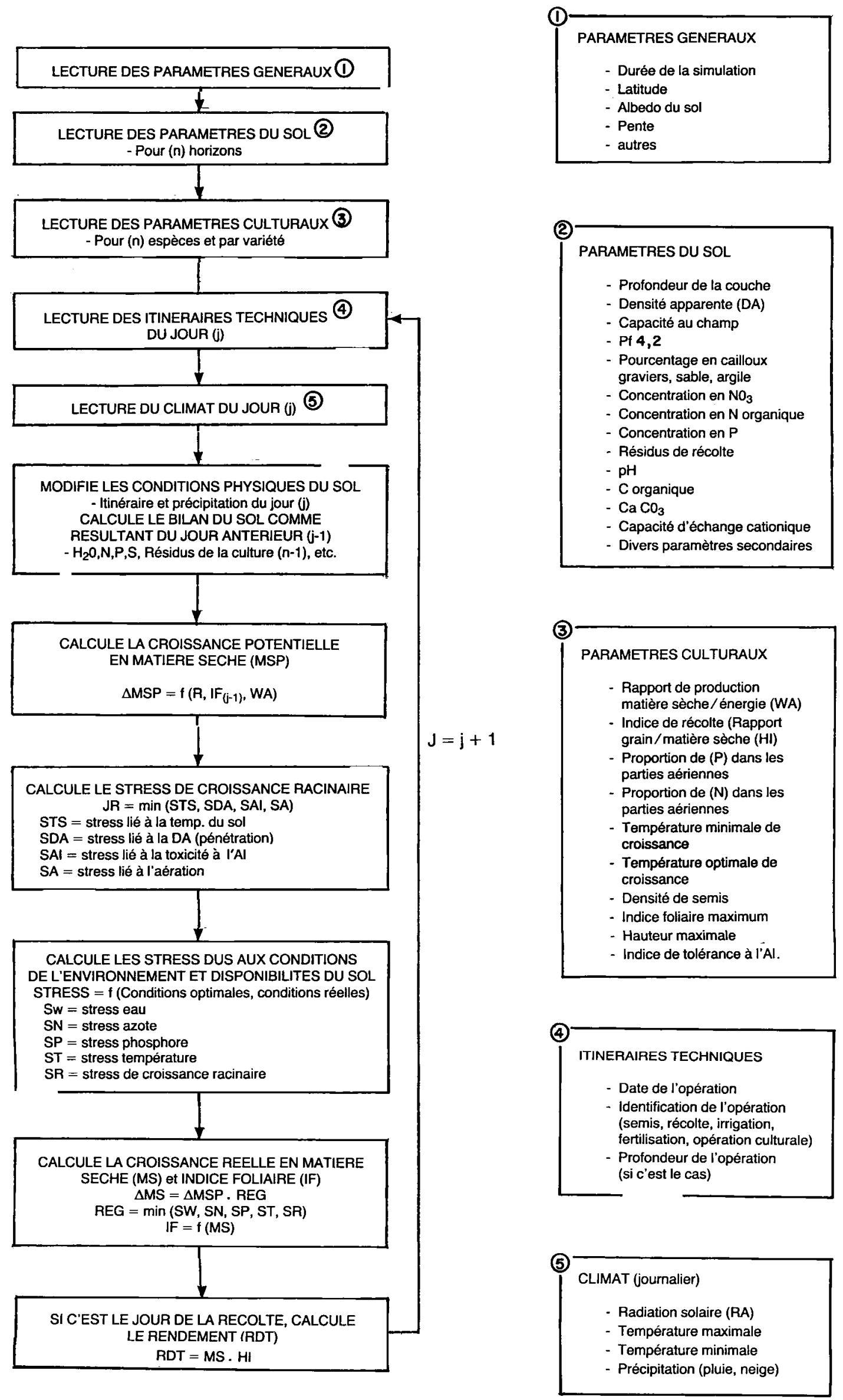

Figure 1

Schéma du principe de fonctionnement du modèle

Diagram of functioning model. 
moins grande activation de la minéralisation des matières organiques fraîches et de l'humus.

\section{RÉSULTATS}

Nous présenterons quelques exemples de simulation de l'évolution pluriannuelle de l'eau et de l'azote nitrique du sol sous quelques rotations et nous les comparerons aux mesures réalisées à différentes périodes. Nous en examinerons ensuite les effets sur l'alimentation hydrique de quelques cultures d'été, et sur la nutrition azotée du blé d'hiver, avec leurs conséquences sur les rendements.

\section{A. Simulation de l'évolution de la consommation en eau, de l'azote nitrique du sol, puis de la matière sèche totale, à l'échelle de la rotation}

A titre d'exemple nous présentons sur les figures 2 et 3 2 rotations courtes aux 3 niveaux d'intensification, l'une sorgho-blé non irriguée, peu intensifiée (intrant I), l'autre maïs-blé irrigué à 2 niveaux d'intensification moyen (intrant II) et élevé (intrant III). Ces niveaux sont différenciés par des objectifs de rendements différents qui mettent en jeu, essentiellement, des quantités d'eau d'irrigation ou de fumure azotée plus ou moins importantes.

On peut observer, figure 2 , que les consommations en eau (ETR) et les déficits du sol sont correctement simulés par le modèle, au cours des 4 années. L'ampleur de ces consommations et de ces déficits est liée aux niveaux d'intensification des rotations. Les mesures hebdomadaires de contrôles hydriques réalisées pendant le cycle végétatif de la culture se placent de façon correcte sur les courbes simulées (sur la figure 2 ne sont représentés que quelques points).

Quant aux nitrates, leur quantité dans le sol s'élève également selon le niveau d'intensification de la rotation; par contre, la précision quantitative paraît moins bonne si l'on admet comme référence la valeur des mesures effectuées: en effet, ces mesures de «l'azote nitrique sortie hiver» sont très fluctuantes dans nos conditions pédoclimatiques (DECAU \& PUJOL, 1981) comme en témoignent les teneurs mini-maxi $(\mathrm{m}-\mathrm{M})$ figurant sur les graphiques. Toutefois, les résultats simulés s'inscrivent dans les limites des mesures réalisées ; par conséquent, la simulation tient compte correctement des résidus de récolte, des reliquats de fumure, et ceci sans dérive apparente sur plusieurs années.

La figure 3 rend compte de l'évolution de la matière sèche totale (MST) au cours des 4 années. L'ajustement entre les valeurs simulées et mesurées est satisfaisant, ce qui confirme globalement la validité des simulations précédentes. A noter que les résidus de récolte simulés sont d'autant plus importants que le niveau d'intensification de la rotation est élevé.

\section{B. Simulation des bilans hydriques annuels}

Les résultats de dessiccation du sol présentés globalement à la figure 2 peuvent être mieux précisés par simulation de profil de dessiccation prenant en compte les différents horizons comme le montre la figure 4. On observe une bonne simulation des profils hydriques depuis la surface avec un écart maximum de 13 p. 100 par rapport aux profils mesurés, et ce pour les mois de juillet et d'août.
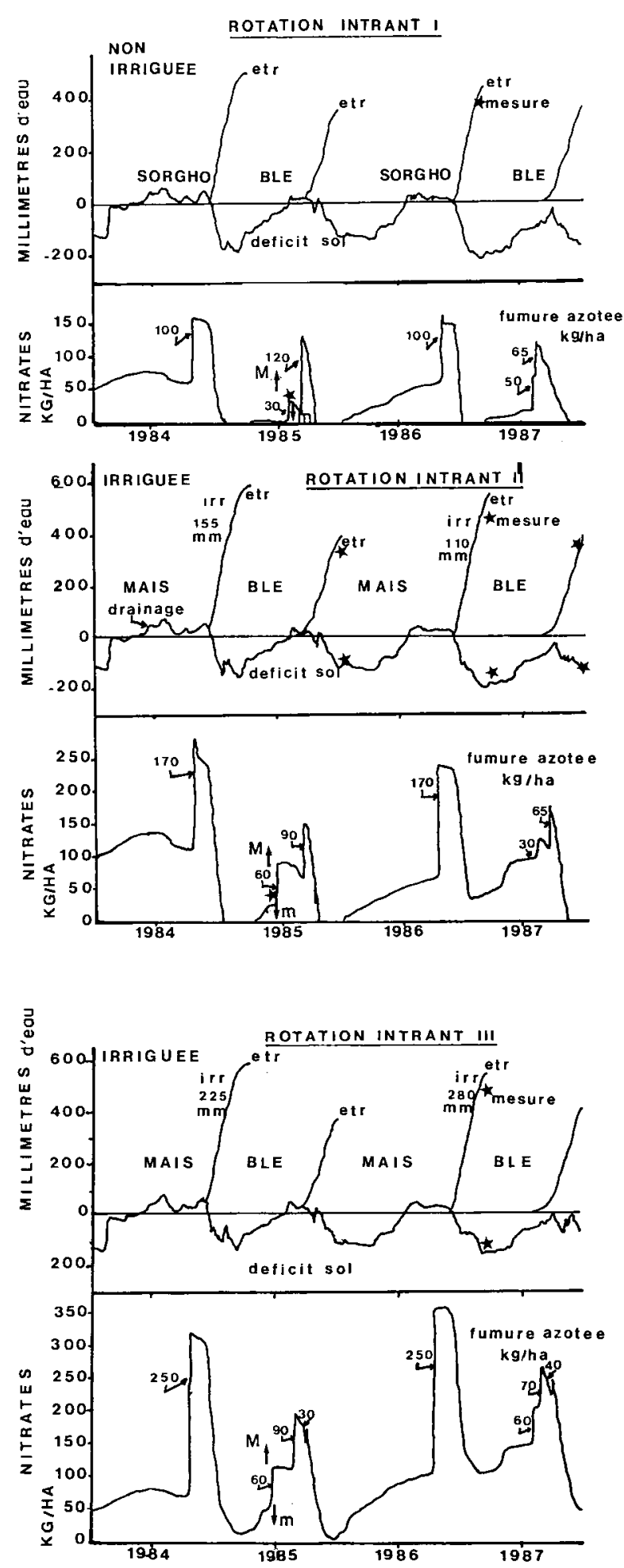

Figure 2

Evolution pluriannuelle des consommations eau, déficits du sol et des nitrates dans le profil selon 3 niveaux d'intensification.

Water consumption, soil water deficit, nitrogen in soil for 3 levels of intensification, over several years. 

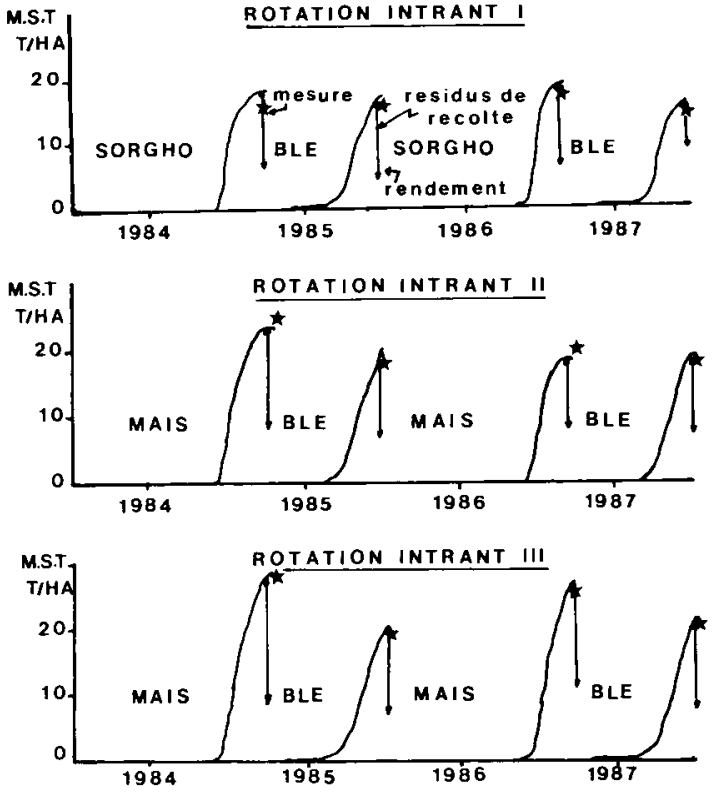

Figure 3

Evolution de la matière sèche totale (M.S.T.) à 3 niveaux d'intrants. Dry matter (M.S.T.) for 3 levels of inputs, over several years.

Ces résultats corrects sont essentiellement dus aux améliorations apportées au sous-modèle eau (CHARPENTEAU et al., 1986) qui tiennent notamment compte des modalités d'extraction de l'eau du sol selon les espèces (Maertens \& Cabelguenne, 1974).

Dans tous les cas, les simulations tiennent compte de "l'héritage» des cultures et techniques réalisées au cours du temps : fumure, irrigation, toute intervention de travail du sol sur la profondeur travaillée, avec leurs effets sur la porosité et l'aération, la température et l'état hydrique du sol, la minéralisation des résidus de récolte et de l'humus, la teneur en $\mathrm{N}$ nitrique, les pertes par drainage.

\section{Simulation des productions annuelles}

Nous examinerons le cas de quelques cultures prises dans diverses rotations conduites à différents niveaux d'intensification.

\section{Cultures d'été}

Le tableau 1 présente les résultats mesurés et simulés des cultures d'été de 1986.

On peut remarquer que le modèle simule correctement la production de matière sèche dans les différentes conditions d'alimentation hydrique et azotée. Par contre, la simulation des rendements est beaucoup moins satisfaisante, notamment en présence de cultures subissant un déficit d'alimentation hydrique. Le modèle utilise un rapport grain/matière sèche totale (différent entre espèces) qui reste pratiquement le même pour chaque culture quel que soit le stress hydrique qu'elle subit. Or, ce rapport évolue pour chaque espèce selon les conditions d'alimentation en eau; par exemple, il est

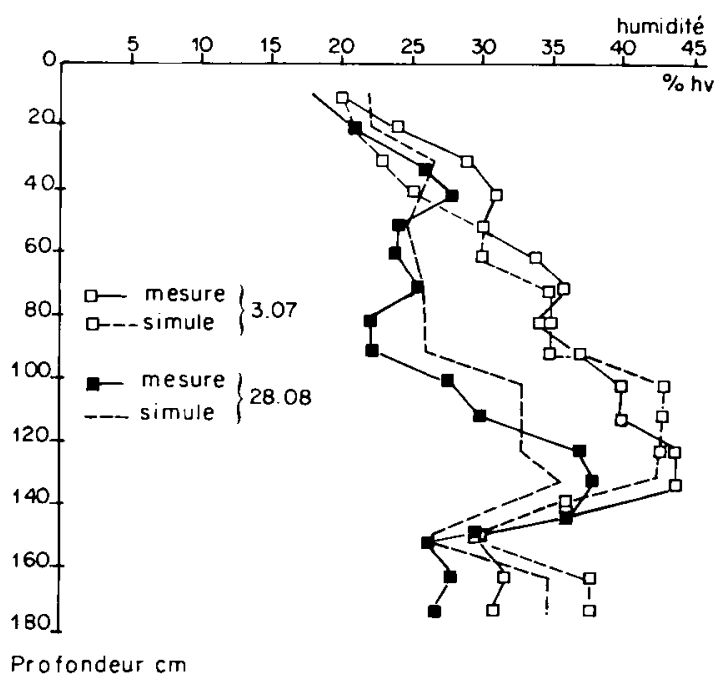

Figure 4

Profils hydriques sous culture de maïs à 2 dates différentes. Comparaison entre mesures et simulations.

Hydric profiles of corn for 2 different dates. Comparison between measured and simulated data.

plus faible pour maïs et tournesol qui subissent un stress hydrique important, ou plus élevé pour le soja lorsque le stress hydrique pendant la période critique est faible. La simulation correcte des rendements exige donc que le modèle prenne en compte l'alimentation hydrique au cours du cycle en simulant une évolution du rapport grain/matière sèche totale.

\section{Cultures d'hiver}

Nous présentons quelques résultats du blé, qui en tant que culture d'hiver, a l'avantage d'être présent dans toutes les rotations avec un large éventail de précédents culturaux et de niveaux d'intensification. Le tableau 2 résume des résultats obtenus en 1985 et 1986. Par contre, on constate à nouveau que les valeurs simulées du stock d'azote dans le sol à la sortie de l'hiver sont éloignées de celles mesurées. Un doute subsiste donc quant à la fiabilité de ces valeurs, mais aussi bien sur les mesures que sur les simulations. Les simulations sont correctes pour la matière sèche totale quels que soient les précédents et les niveaux d'intensification, mais moins bonnes pour les rendements, généralement plus faibles que les rendements mesurés, notamment en l'absence de fumure azotée. En effet, le modèle utilise dans tous les cas un rapport grain/matière sèche totale de 0,39 alors que ce rapport est voisin de $0,43 \pm 0,02$ avec une fumure azotée appropriée à l'objectif de rendement et tenant compte du précédent, et de $0,49 \pm 0,02$ en l'absence de fumure azotée. La modification de ce rapport apporte une nette amélioration de la simulation du rendement. En l'absence de fumure azotée, on peut noter l'importance du stress azote après sorgho et maïs dans le niveau II d'intensification, à reliquats modérés, alors que ce stress est plus faible après féverole et soja ainsi qu'après colza à reliquats $\mathrm{N}$ plus importants. Avec fumure azotée, le stress d'alimentation azotée a fortement diminué, il reste cependant important après tournesol où la fumure azotée a été insuffisante par erreur. 
TABLEAU 1

Synthèse de résultats mesurés ( $M$ )-simulés ( $S$ ) des 4 cultures d'été (année 1986).

Comparison of measured $(M)$ and simulated (S) results of 4 summer crops (1986).

\begin{tabular}{|c|c|c|c|c|c|c|c|c|c|c|c|c|c|}
\hline & & & MAÏS & & & SOJA & & & UURNESC & & & SORGHO & \\
\hline & & $\begin{array}{c}\text { non } \\
\text { irrigué }\end{array}$ & $\begin{array}{l}\text { irrigué } \\
\text { période } \\
\text { critique }\end{array}$ & $\begin{array}{l}\text { irrigué } \\
\text { intensif }\end{array}$ & $\begin{array}{l}\text { non } \\
\text { irrigué }\end{array}$ & $\begin{array}{l}\text { irrigué } \\
\text { période } \\
\text { critique }\end{array}$ & $\begin{array}{l}\text { irrigué } \\
\text { intensif }\end{array}$ & $\begin{array}{l}\text { non } \\
\text { irrigué }\end{array}$ & $\begin{array}{l}\text { irrigué } \\
\text { période } \\
\text { critique }\end{array}$ & $\begin{array}{l}\text { irrigué } \\
\text { intensif }\end{array}$ & $\begin{array}{c}\text { non } \\
\text { irrigué }\end{array}$ & $\begin{array}{l}\text { irrigué } \\
\text { période } \\
\text { critique }\end{array}$ & $\begin{array}{l}\text { irrigué } \\
\text { intensif }\end{array}$ \\
\hline Irrigation & $(\mathrm{mm})$ & 0 & 110 & 280 & 0 & 100 & 190 & 0 & 100 & 180 & 0 & 80 & 200 \\
\hline Fumure azotée & (kg N/ha) & 100 & 170 & 250 & 0 & 0 & 0 & 50 & 130 & 170 & 100 & 150 & 190 \\
\hline $\begin{array}{l}\text { Consommation } \\
\text { mm } \\
\text { M.S.T. } \\
\text { q.ha }\end{array}$ & $\begin{array}{l}M \\
S \\
M / S \\
M \\
S \\
M / S\end{array}$ & $\begin{array}{r}295 \\
287 \\
1,03 \\
109 \\
101 \\
1,08\end{array}$ & $\begin{array}{r}379 \\
455 \\
0,83 \\
172 \\
148 \\
1,16\end{array}$ & $\begin{array}{r}454 \\
524 \\
0,87 \\
252 \\
223 \\
1,13\end{array}$ & $\begin{array}{r}396 \\
347 \\
1,14 \\
63 \\
68 \\
0,93 \\
\end{array}$ & $\begin{array}{r}426 \\
380 \\
1,12 \\
85 \\
75 \\
1,13\end{array}$ & $\begin{array}{r}500 \\
481 \\
1,04 \\
112 \\
108 \\
1,04\end{array}$ & $\begin{array}{r}376 \\
311 \\
1,21 \\
127 \\
105 \\
1,21\end{array}$ & $\begin{array}{r}525 \\
408 \\
1,29 \\
139 \\
139 \\
1,00\end{array}$ & \begin{tabular}{r|}
534 \\
452 \\
1,18 \\
142 \\
160 \\
0,89
\end{tabular} & $\begin{array}{r}334 \\
336 \\
1,29 \\
114 \\
124 \\
0,92\end{array}$ & $\begin{array}{r}410 \\
348 \\
1,18 \\
185 \\
179 \\
1,03\end{array}$ & $\begin{array}{r}441 \\
435 \\
1,01 \\
222 \\
212 \\
0,98\end{array}$ \\
\hline $\begin{array}{l}\text { Rendement } \\
\text { Grain sec } \\
\text { q.ha } \\
\text { Grain/MST }\end{array}$ & $\begin{array}{l}M \\
S \\
M / S \\
M \\
S\end{array}$ & $\begin{array}{r}26 \\
44 \\
0,59 \\
0,24 \\
0,44\end{array}$ & $\begin{array}{r}83 \\
68 \\
1,22 \\
0,48 \\
0,46\end{array}$ & $\begin{array}{r}115 \\
106 \\
1,08 \\
0,46 \\
0,48\end{array}$ & $\begin{array}{r}18 \\
19 \\
0,94 \\
0,29 \\
0,28\end{array}$ & $\begin{array}{r}29 \\
22 \\
1,32 \\
0,34 \\
0,29\end{array}$ & $\begin{array}{r}31 \\
31 \\
1,00 \\
0,28 \\
0,29\end{array}$ & $\begin{array}{r}29 \\
23 \\
1,26 \\
0,23 \\
0,22\end{array}$ & $\begin{array}{r}43 \\
31 \\
1,39 \\
0,31 \\
0,22\end{array}$ & $\begin{array}{r}38 \\
36 \\
1,06 \\
0,27 \\
0,23\end{array}$ & $\begin{array}{r}66 \\
57 \\
1,16 \\
0,58 \\
0,46\end{array}$ & $\begin{array}{r}95 \\
84 \\
1,13 \\
0,51 \\
0,47\end{array}$ & $\begin{array}{r}100 \\
100 \\
1,00 \\
0,45 \\
0,47\end{array}$ \\
\hline
\end{tabular}

TABLEAU 2

Résultats mesurés $M$ - simulés $S$ - des blés 1985-1986 et 1984-1985().

Measured $M$ and simulated $S$ results for 1985-1986 and 1984-1985, () winter wheat.

\begin{tabular}{|c|c|c|c|c|c|c|c|c|c|c|c|}
\hline \multicolumn{2}{|l|}{$\begin{array}{l}\text { Précédents } \\
\text { culturaux }\end{array}$} & \multicolumn{2}{|c|}{$\begin{array}{c}\text { Soja } \\
\text { Non IRR }\end{array}$} & $\begin{array}{l}\text { Soja } \\
\text { IRR }\end{array}$ & \multicolumn{2}{|c|}{$\begin{array}{l}\text { Maïs } \\
\text { IRR }\end{array}$} & \multicolumn{2}{|c|}{\begin{tabular}{l}
\multicolumn{2}{c}{ Sorgho } \\
N. IRR
\end{tabular}} & \multirow{2}{*}{$\begin{array}{l}\text { Tournes. } \\
\text { N. IRR } \\
\text { II }\end{array}$} & \multirow{2}{*}{$\begin{array}{c}\text { Colza } \\
\text { III }\end{array}$} & \multirow{2}{*}{$\begin{array}{c}\text { Féverole } \\
\text { II }\end{array}$} \\
\hline \multicolumn{2}{|c|}{$\begin{array}{c}\text { Niveau } \\
\text { d'intensification } \\
\text { de la rotation (1) }\end{array}$} & I & II & III & II & III & II & II & & & \\
\hline \multicolumn{12}{|c|}{ 1) Avec fumure minérale azotée } \\
\hline $\begin{array}{l}\text { N fumure } \mathrm{kg} / \mathrm{ha} \\
\text { du blé }\end{array}$ & & $\frac{90}{-}$ & $\begin{array}{r}140 \\
(140)\end{array}$ & $\frac{170}{-}$ & $\begin{array}{r}150 \\
(150)\end{array}$ & $\begin{array}{r}200 \\
-\end{array}$ & $\begin{array}{r}200 \\
(200)\end{array}$ & $\begin{array}{r}210 \\
(200)\end{array}$ & $\frac{80}{-}$ & $\frac{150}{-}$ & $\begin{array}{r}90 \\
(60)\end{array}$ \\
\hline $\begin{array}{l}\text { N. NO3-sol } \\
\text { avant fumure }\end{array}$ & $\begin{array}{l}\mathrm{M} \\
\mathrm{S}\end{array}$ & $\overline{74}$ & $\begin{array}{r}130 \\
67\end{array}$ & $\begin{array}{l}60 \\
93\end{array}$ & $\begin{array}{l}94 \\
48\end{array}$ & $\overline{86}$ & $\begin{array}{r}108 \\
10\end{array}$ & $\begin{array}{l}62 \\
34\end{array}$ & $\overline{29}$ & $\overline{119}$ & $\begin{array}{l}184 \\
126\end{array}$ \\
\hline $\begin{array}{l}\text { Production } \\
\text { MST q/ha }\end{array}$ & $\begin{array}{l}\mathrm{M} \\
\mathrm{M} / \mathrm{S}\end{array}$ & $\frac{145}{0,96}$ & $\begin{array}{r}157 \\
(162) \\
0,99 \\
(0,99)\end{array}$ & $\frac{182}{1,09}$ & $\begin{array}{r}166 \\
(140) \\
1,04 \\
(0,80)\end{array}$ & $\frac{176}{1,22}$ & $\begin{array}{r}170 \\
(154) \\
0,87 \\
(0,86)\end{array}$ & $\begin{array}{l}168 \\
1,04 \\
-\end{array}$ & $\begin{array}{l}146 \\
-\end{array}$ & $\begin{array}{l}146 \\
- \\
-\end{array}$ & $\begin{array}{r}175 \\
155 \\
(0,84)\end{array}$ \\
\hline $\begin{array}{l}\text { Rendement } \\
\text { grain sec } \mathrm{q} / \mathrm{ha}\end{array}$ & $\begin{array}{l}\mathrm{M} \\
\mathrm{M} / \mathrm{S}\end{array}$ & $\frac{62}{1,07}$ & $\begin{array}{r}69 \\
(72) \\
1,08\end{array}$ & $\frac{75}{1,15}$ & $\begin{array}{r}73 \\
(68) \\
1,18\end{array}$ & $\frac{72}{1,11}$ & $\begin{array}{r}74 \\
(67) \\
1,21\end{array}$ & $\frac{73}{1,16}$ & $\frac{66}{1,40}$ & $\frac{64}{0,98}$ & $\frac{77}{1,18}$ \\
\hline $\begin{array}{l}\text { Grain/MST } \\
(\mathrm{S}=0,39) \\
\text { Stress azote }(2)\end{array}$ & $\begin{array}{l}M \\
S\end{array}$ & $\frac{0,43}{8}$ & $\begin{array}{r}0,44 \\
(0,44) \\
14\end{array}$ & $\frac{0,41}{12}$ & $\begin{array}{r}0,44 \\
(0,48) \\
14\end{array}$ & $\frac{0,41}{12}$ & $\begin{array}{r}0,44 \\
(0,44) \\
13\end{array}$ & $\begin{array}{r}0,43 \\
(0,39) \\
12\end{array}$ & $\frac{0,45}{32}$ & $\frac{0,44}{11}$ & $\begin{array}{r}0,44 \\
(0,41) \\
10\end{array}$ \\
\hline \multicolumn{12}{|c|}{ 2) Sans fumure minérale azotée } \\
\hline $\begin{array}{l}\text { Production } \\
\text { MST q/ha }\end{array}$ & $\begin{array}{l}\mathrm{M} \\
\mathrm{M} / \mathrm{S}\end{array}$ & $\begin{array}{r}87 \\
0,97\end{array}$ & $\begin{array}{r}89 \\
1,13\end{array}$ & $\begin{array}{r}114 \\
1,06\end{array}$ & $\begin{array}{r}99 \\
1,32\end{array}$ & $\begin{array}{r}107 \\
0,99\end{array}$ & $\begin{array}{r}74 \\
1,72\end{array}$ & $\begin{array}{r}68 \\
0,92\end{array}$ & - & $\begin{array}{r}114 \\
0,97\end{array}$ & $\begin{array}{l}100 \\
0,78\end{array}$ \\
\hline $\begin{array}{l}\text { Rendement } \\
\text { grain sec } \mathrm{q} / \mathrm{ha}\end{array}$ & $\begin{array}{l}\mathrm{M} \\
\mathrm{M} / \mathrm{S}\end{array}$ & $\begin{array}{r}41 \\
1,17\end{array}$ & $\begin{array}{r}51 \\
1,50\end{array}$ & $\begin{array}{r}57 \\
1,33\end{array}$ & $\begin{array}{r}47 \\
1,62\end{array}$ & $\begin{array}{r}52 \\
1,24\end{array}$ & $\begin{array}{r}36 \\
2,12\end{array}$ & $\begin{array}{r}37 \\
1,28\end{array}$ & - & $\begin{array}{r}55 \\
1,20\end{array}$ & $\begin{array}{r}48 \\
0,96\end{array}$ \\
\hline $\begin{array}{l}\text { Grain/MST } \\
(\mathrm{S}=0,39)\end{array}$ & $\mathbf{M}$ & 0,47 & 0,52 & 0,50 & 0,47 & 0,49 & 0,49 & 0,54 & - & 0,48 & 0,48 \\
\hline Stress azote (2) & $\mathrm{S}$ & 41 & 39 & 33 & 58 & 39 & 83 & 55 & - & 31 & 22 \\
\hline
\end{tabular}

(1) I faible - II moyen - III fort

(2) Nombre de jours de stress $x$ intensité journalière du stress. 


\section{DISCUSSION}

Les premières améliorations apportées à la version originale du modèle EPIC, fournissent des simulations proches des valeurs mesurées pour la matière sèche totale, mais qui diffèrent quelquefois pour la simulation des rendements, alors que les aspects hydriques pour le sol et la plante et les nitrates dans le profil du sol sont actuellement satisfaisants à quelques aménagements près.

Nous avons montré que ce type de modèle ne tient pas compte de l'effet de stress hydrique et/ou azote sur l'élaboration du rendement, et notamment sur l'évolution du rapport grain/matière sèche totale. Dans un premier temps, une nouvelle version en cours d'élaboration corrigera, pour les différentes cultures, la valeur du rapport grain/matière sèche en tenant compte de stress subis entre les principaux stades phénologiques. Ceci est particulièrement important chez les oléagineux où la transformation de l'énergie en biomasse varie selon les phases du cycle et induit une modification du rapport grain/matière sèche totale. Ce travail est actuellement en cours chez le tournesol en distinguant 4 phases végétatives (juvénile, croissance, floraison et remplissage des graines) que le modèle reconnaîtra par la somme des températures correspondant à chaque phase et où seront introduits les effets de stress eau et/ou azote et leurs conséquences en fin de culture sur l'indice de récolte, donc sur le rendement. Cette démarche sera étendue par la suite aux autres cultures d'été.

Un autre défaut du modèle concerne le paramètre plante relatif à la forme et à l'agressivité de l'enracinement qui conditionne les possibilités d'extraction de l'eau du sol plus ou moins profondément et intensément. Une modification de ces paramètres et des équations correspondantes pourrait aussi améliorer les simulations de matière sèche totale, en condition non irriguée du soja, du tournesol et du sorgho (tabl. 1).

Pour le blé qui subit un faible stress hydrique et lorsque l'azote n'a pas été limitant, les paramètres plantes (qui ont été validés séparément) liés à la transformation de l'énergie lumineuse en biomasse, l'indice foliaire maximum, le pourcentage d'utilisation d'unités de chaleur au-delà duquel la surface foliaire diminue, semblent corrects alors que le rapport grain/matière sèche totale demande à être précisé. Dans nos conditions, ce rapport quoique très global, peut intégrer dans une $1^{\mathrm{re}}$ approche à la fois l'effet densité en épis et nombre de grains sensibles à la nutrition azotée, et le poids d'un grain particulièrement affecté par des stress thermiques en cours de maturation qui raccourcissent fortement le palier hydrique du grain et dont le modèle ne tient pas compte.

Pour la simulation de l'azote nitrique disponible pour les cultures, on a observé sous blé (les mesures ne sont réalisées que sous blé en hiver) quelques divergences entre mesures et simulations (fig. 2 et tabl. 2) probablement liées aux difficultés de contrôle du $\mathrm{N}$ nitrique du sol à cette époque (DECAU \& PUJOL, 1981). Toutefois, le calcul de la fumure azotée du blé à partir des résultats de simulation et compte tenu du niveau d'intrant, a permis d'obtenir un comportement sensiblement normal du blé. Les écarts de valeurs, observés entre les mesures et la simulation, proviennent de la difficulté de contrôle de l'azote nitrique du sol et ainsi que des grandes variations de ces teneurs à intervalles très courts à cette époque, alors que le modèle simule une évolution continue de cette teneur à partir de : la teneur de $\mathrm{C}$ organique, de l'état hydrique du sol, des résidus de culture des années précédentes, des processus de minéralisation, de réorganisation, de dénitrification et de l'entraînement par lessivage. Nous n'avons pu encore valider chacun de ces points pris isolément. On pourrait cependant perfectionner le sous modèle azote-matière organique en utilisant, en partie, des modèles plus spécifiques qui simulent l'évolution de l'azote organique de manière plus précise (MOLINA et al., 1983; CLAY et al., 1985a et $b$; DEANS et al., 1986).

\section{CONCLUSIONS}

Dans sa version améliorée, ce modèle peut être utilisé à la fois comme outil de recherche et de développement et peut être généralisé à diverses situations sous réserve d'adapter quelques paramètres aux conditions spécifiques à ces situations :

- en tant qu'outil de recherche en mettant en évidence des effets difficiles à quantifier ou en simulant des conditions difficiles à tester, ou non expérimentées (BlanCHET et al., 1988a et $b$ ). Il fournit aussi aux économistes un moyen de simuler et de prendre en compte l'impact de diverses techniques coûteuses sur la production ou les effets d'années climatiques différentes ;

- en tant qu'outil d'aide à la décision il fournit des données permettant de conduire l'irrigation (CHARPENTEAU \& Williams, 1986) ou de calculer la fumure azotée (par exemple simulation de la quantité d'azote sortie hiver). Des précisions à court ou moyen terme pourraient être obtenues dans ces domaines en utilisant EPIC en temps réel, c'est-à-dire en arrêtant une simulation un jour donné, de réaliser des corrections éventuelles de paramètres d'état du système sol-plante au vu d'observations au champ ou avec le climat subi, et puis de faire des prévisions en simulant le climat ultérieur grâce au générateur de climat (CHARPENTEAU, 1987).

Au cours de 4 années de simulation, on peut constater que dans sa conception actuelle, EPIC peut être utilisé comme modèle de fonctionnement de successions de cultures et de diagnostic de contraintes et comme voie complémentaire intéressante de l'expérimentation. Il fournit en effet à chaque instant et pour un coût modéré des informations sur les états du système plante-solclimat-technique-successions de cultures, non directement observables (ChARPENTEAU, 1987).

Il peut aussi être utilisé en tant que modèle exploratoire de situations pédoclimatiques différentes en simulant le comportement des cultures insérées dans diverses rotations conduites à différents niveaux d'intensification.

C'est aussi pour les agronomes une possibilité de synthèse des connaissances couvrant un large champ, qui nécessite une réflexion et un travail collectif associant divers spécialistes. Ceci permet d'établir de meilleurs diagnostics et une meilleure gestion des phénomènes complexes auxquels on se trouve confronté lors de l'étude du fonctionnement de rotations culturales. En 
même temps, le modèle peut et doit être amélioré comme cela a été fait déjà par la simulation de la fixation symbiotique de l'azote pour le soja, et le sera pour le pois et la féverole.

L'ensemble des améliorations constitue un des prochains axes de recherches qui doit déboucher sur un autre modèle, ARMAGNAC - Agricultural Research Management Alternatives Given Numerical Assessment Criteria - en collaboration avec nos partenaires américains. Des modèles annuels conçus par espèce pourront probablement nous aider dans cette démarche.

Reçu le 10 juillet 1987 Accepté le 24 avril 1988.

\section{REMERCIEMENTS}

Ces études sont conduites avec la collaboration de J.L. Charpenteau, du Laboratoire de Biométrie, que nous remercions pour son aide et ses conseils.

\section{RÉFÉRENCES BIBLIOGRAPHIQUES}

Blanchet R., Bouniols A., Gelfi N., Cabelguenne M., Desclaux D., Bona S., 1988a. Simulation des principales contraintes agronomiques limitant le rendement du soja dans différentes situations françaises. I. Le modèle et sa validation. C. R. Acad. Agric. Fr., 74 (séance du 16 mars 1988) (sous presse).

Blanchet R., Desclaux D., Gelfi N., Quinones H., Bosc N., $1988 b$. Simulation des principales contraintes agronomiques limitant le rendement du soja dans différentes situations françaises. II. Exploration de situations variées. C. R. Acad. Agric. Fr., 74 (séance du 16 mars 1988) (sous presse).

Cabelguenne M., Charpenteau J.-L., Jones C. A., Marty J.-R., Fiellier J.-P., 1986. Conduite des systèmes de grandes cultures et prévisicn des rendements : tentatives de modélisation. II. - Etalonnage du modèle : résultats et perspectives. C. $R$. Acad. Agric. Fr., 72 (2), 125-132, séance du 29 janvier 1986.

Charpenteau J.-L., Jones C. A., Marty J.-R., Rellier J.-P., Williams J. R., 1986. Conduite des systèmes de grandes cultures et prévision de rendements : tentative de modélisation. I. - Choix et construction du modèle. C. R. Acad. Agric. Fr., 72 (1), 118-124, séance du 29 janvier 1986.

Charpenteau J.-L., 1987. Les modèles de simulation et leur utilisation pour l'aide à la décision en agriculture. In Modèles et aide à la décision agricole. Colloque Toulouse (Fr.), 13 janvier 1987, 54 p.

Charpenteau J.-L., Williams J. R., 1986. Using a simulation model for irrigation scheduling in cropping system study: first results. C.E.E. Workshop Agricultural Water Management. Leuven, 25-28 nov. Belgium, $12 \mathrm{p}$.

Clay D. E., Clapp C. E., Molina J. A. E., Linden D. R., 1985a. Nitrogen tillage residue management. I. - Simulating soil and plant behavior by the model NCSWAP. Plant Soil, 84, 67-77.

Clay D. E., Molina J. A. E., Clapp C. E., Linden D. R., $1985 b$. Nitrogen tillage residue management. II. - Calibration of potential rate of nitrification by model simulation. Soil. Sci. Soc. Am. J., 49, 322-325.

Deans J. R., Molina J. A. E., Clapp C. E., 1986. Models for producting potentially mineralizable nitrogen and decomposition rate constant. Soil Sci. Am. J., 50, 323-326.

Decau J., Pujol B., 1981. Difficulté d'application d'une méthode de prévision de la fertilisation azotée des blés d'hiver dans le Sud-Ouest de la France. C.E.E. Division VI F4 in "Devenir de l'azote dans la fertilisation $N$ des blés d'hiver. Influence de l'alimentation hydrigue ». Séminaire Toulouse, France, 216-221.

Jackson B. S., Arkin G. F., 1982. Fruit growth in a cotton simulation model. Texas Agricultural Experiment Station Temple. The Beltwide cotton conference, Las Vegas, 61-64.
Jones C. A., Ritchie J. T., Kiniry J. R., Godwin D. C., Otter S. I., 1984 The CERES wheat and maize models. In ICRISAT (International Crops Research Institute for the Semi-Arid Tropics). Proc. International Symp. on minimum data Sets for Agrotechnology Transfer. March 21-26, 1983, 95-100. Pantancheru, India : ICRISAT Center.

Jones C. A., Kiniry J. R., 1986. CERES-MAIZE a simulation model of maize growth and development. Texas ARM. University Press. College Station, $194 \mathrm{p}$.

Maertens C., Cabelguenne M., 1974. Intensité et limite de dessèchement du sol en relation avec l'enracinement de quelques espèces végétales cultivées. C. R. Acad. Sci., Sér. D : Sci. nat., 279, 2039-2042 (23 décembre 1974).

Maertens C., 1987. Ways of Wing endoscopy to determine growth and quality of root systems. American Society of Agronomy, Crop Science Society of America, and Soil Science Society of America, $677 \mathrm{~S}$. SEGOE Rd; Madison, W I 53711 U.S.A. Minirhizotron observation Tubes: Methods and applications for measuring rhizosphere dynamics, A.S.A. special publication $\mathrm{n}^{\circ} 501,37$.

Marty J.-R., Hutter W., Rellier J.-P., 1981. Projet de programme de recherches expérimentales sur l'optimisation des intrants dans des rotations avec céréales et oléoprotéagineux avec ou sans irrigation. In Séminaire C.E.E., 15-16 janvier 1981, Toulouse, 119-131.

Molina J. A. E., Clapp C. E., Chaffer M. J., Chichester F. W., Larson W. E., 1983. NCSOIL, a model of nitrogen and carbon transformation in soil : description, calibration and behavior, Soil Sci. Am. J. Soc., 47, 85-91.

Pearson C. J., Steer B. T., Coaldrake P. D., 1985. A preliminary model to predict growth and oil yield of sunflower Hysun 30. Actas $X I$. Conferencia internationale de girasol. Mar del Plata, Argentine, vol. $1,177-182$.

Sebillotte M., 1974. Agronomie et Agriculture. Essai d'analyse des tâches de l'agronome. Cah. O.R.S.T.O.M., Ser. Biol.. 24, 3-25.

Whisler F. D., Acok B., Baker D. N., Fye R. E., Hodges H. F., Lambert J. R., Lemmon H. E., McKinion J. M., Reddy V. R., 1986. Crop simulation models in agronomic systems. Adv. Agron., 40, 141-208.

Wilkinson G. C., Jones J. N., Boote K. J., Ingram K. T., Mishoe M., 1983. Modeling soybean growth for crop management. Trans. A.S.A.E., 26, 63-73.

Richardson C. W., 1981. Stochastic simulation of daily precipitation, temperature and solar radiation. Water Resour. Res., 17, 182-190.

Williams J. R., Jones C. A., Dyke P. T., 1984. A modeling approach to determining the relation between erosion and soil productivity. Trans. A.S.A.E., 27, 129-144. 